\title{
eHealth in kidney care
}

Chia-shi Wang $\mathbb{1}^{1,2} \bowtie$ and Elaine $K \mathrm{u}^{3}$

eHealth is gaining momentum in nephrology, although evidence for its efficacy remains unclear and challenges to its widespread adoption persist. Successful integration of eHealth into kidney care will require patient engagement to develop effective interventions and issues such as data validity, regulation, oversight and adequate infrastructure to be addressed.

Globally, $>90 \%$ of the population is within reach of a mobile phone signal and internet service, offering realtime communication across geographical barriers ${ }^{1}$. eHealth, defined as "health services and information delivered or enhanced through the internet and related technologies," ${ }^{2}$ has the potential to extend kidney care beyond traditional episodic care in ambulatory or inpatient settings to care that is integrated into daily life (TABLE 1). The current public health emergency caused by COVID-19 brings new urgency to the integration of eHealth into patient care as the global community grapples with the need to limit direct face-to-face contact at health-care facilities to decrease the risk of virus transmission.

\section{Momentum for eHealth in nephrology}

The potential of eHealth to transform kidney disease screening and management is increasingly recognized. In the USA, the National Kidney Disease Education Program of the National Institute of Diabetes and Digestive and Kidney Diseases (NIDDK) has established a Health Information Technology Working Group to facilitate the identification and management of patients with chronic kidney disease (CKD) using electronic health records (EHRs) (see Related links). In 2019, the group published a validated electronic phenotype for CKD identification using EHRs at five sites for $>2$ million patients ${ }^{3}$.

Numerous health systems have adopted eHealth to improve kidney care. For example, the US Veteran Affairs (VA) health system recognized that a third of veterans with advanced CKD did not receive nephrology care before starting dialysis. To address this care gap, the VA has developed a portfolio of eHealth solutions: a provider-to-provider real-time video consultation service; patient-provider video visits for patients in rural areas; and individually tailored disease monitoring and education via video visits, web-modules and wireless devices such as digital blood pressure monitors and scales ${ }^{4}$.

Policy support for eHealth services is also gaining momentum. In the USA, the 2018 Bipartisan Budget Act changed Medicare policy to allow payments for 2-monthly comprehensive telehealth visits (using interactive video and audio telecommunication) per quarter for all patients receiving home dialysis. These visits can be conducted from patients' homes ${ }^{5}$. Beyond nephrology, legislation on telehealth is rapidly changing in response to COVID-19 to facilitate social distancing and quarantine efforts.

\section{Efficacy of eHealth interventions}

With the rapid growth and momentum of eHealth, one of the most pressing concerns is that its efficacy in kidney care remains unclear. A 2019 Cochrane review concluded that overall, the evidence for eHealth interventions is of low quality and insufficient to guide clinical practice in kidney disease ${ }^{6}$. Moreover, a randomized trial that included 601 patients with CKD found that an eHealth intervention that used home monitoring devices to obtain biometric data (for example, blood pressure and weight) and deliver multidisciplinary care did not reduce mortality, hospitalization rates, emergency department visits or nursing home admissions when compared with usual care ${ }^{4}$. However, patient acceptance and engagement with the eHealth intervention was high; $91 \%$ of participants completed 1 year of follow-up with an average of 14.2 completed virtual visits. On average, participants took 14.9 blood pressure measurements per month and used the educational modules on 5.8 days per month ${ }^{4}$. Multiple other studies have reported high participant satisfaction with kidney eHealth interventions for a variety of reasons, including ease of use, low burden and increased frequency and quality of interactions with health-care staff ${ }^{6}$.

A study of commercially available mobile applications (apps) for kidney care reported that only 7 of 28 were rated as high in quality by both nephrologists and patients. Importantly, patient ratings of app quality correlated poorly with provider ratings, highlighting the importance of incorporating patient input into eHealth solutions. The TAKE-IT trial assessed the efficacy of a multimodal intervention to promote medication adherence that incorporated patient input in the eHealth component $^{8}$. The investigators randomly assigned 189 adolescent and young adult kidney transplant recipients 
Table 1 | Health categories and applications in kidney care

\begin{tabular}{|c|c|c|}
\hline Category & Description & Applications in kidney disease \\
\hline \multirow[t]{2}{*}{$\begin{array}{l}\text { Electronic } \\
\text { health } \\
\text { records }\end{array}$} & $\begin{array}{l}\text { Digital versions of patient medical records that may contain } \\
\text { demographic information, diagnoses, biometric and vital sign } \\
\text { measurements, treatment notes, medication information, } \\
\text { laboratory and radiology results, and billing information }\end{array}$ & $\begin{array}{l}\text { Provider alerts and decision aids for management of AKI and CKD } \\
\text { Screening for CKD } \\
\text { Identification of potential candidates for research and clinical trials }\end{array}$ \\
\hline & $\begin{array}{l}\text { Electronic health records can be accessed by patients or providers } \\
\text { through portals with functions to support care-related activities } \\
\text { (for example, place orders, make appointments) }\end{array}$ & $\begin{array}{l}\text { Engagement of patients in self-management by tracking and } \\
\text { presenting data and providing individualized recommendations }\end{array}$ \\
\hline \multirow[t]{6}{*}{ Telehealth } & \multirow{6}{*}{$\begin{array}{l}\text { The use of digital devices and applications such as video } \\
\text { conferencing and digital stethoscopes to allow interactions } \\
\text { between providers and patients and bridge geographical barriers }\end{array}$} & Virtual visits for patients with CKD in rural areas \\
\hline & & $\begin{array}{l}\text { Multidisciplinary care visits for patients with multiple providers } \\
\text { from different disciplines }\end{array}$ \\
\hline & & Video consultations with nephrologists for primary care providers \\
\hline & & $\begin{array}{l}\text { Discharge follow-up after procedures such as PD catheter } \\
\text { placement }\end{array}$ \\
\hline & & $\begin{array}{l}\text { Replacement of in-person visits in prospective studies and clinical } \\
\text { trials to minimize participant burden }\end{array}$ \\
\hline & & $\begin{array}{l}\text { Urgent visits with providers for patients on dialysis in outpatient } \\
\text { facilities with acute issues (for example, cloudy PD fluid) }\end{array}$ \\
\hline \multirow{7}{*}{$\begin{array}{l}\text { Mobile } \\
\text { health }\end{array}$} & \multirow{2}{*}{$\begin{array}{l}\text { The use of mobile devices such as personal digital assistants, } \\
\text { mobile phones, tablets, smartphones, and wearables (for example, } \\
\text { watches, skin patches) to provide medical care }\end{array}$} & Mobile app to check medication safety for patients with CKD \\
\hline & & Educational app for care after transplantation \\
\hline & \multirow[t]{5}{*}{ Methods of care provision include text messaging and mobile apps } & $\begin{array}{l}\text { Smartphone camera read of urine test strips for urinary tract } \\
\text { infection or proteinuria detection }\end{array}$ \\
\hline & & $\begin{array}{l}\text { Blood pressure monitoring and transmission of data to health-care } \\
\text { providers }\end{array}$ \\
\hline & & $\begin{array}{l}\text { Mobile app for dietary monitoring (for example, salt and potassium } \\
\text { intake) }\end{array}$ \\
\hline & & $\begin{array}{l}\text { Electronic pillboxes for medication adherence monitoring } \\
\text { and reminders }\end{array}$ \\
\hline & & Fluid overload monitoring via bioimpedance sensors \\
\hline \multirow[t]{2}{*}{ Web app } & \multirow{2}{*}{$\begin{array}{l}\text { A programme delivered through a web browser } \\
\text { to internet-connected devices such as computers and } \\
\text { smartphones }\end{array}$} & Educational modules for patients with CKD \\
\hline & & $\begin{array}{l}\text { Online cognitive behavioural therapy for patients on dialysis } \\
\text { who have depression }\end{array}$ \\
\hline \multirow{2}{*}{$\begin{array}{l}\text { Social } \\
\text { media }\end{array}$} & \multirow{2}{*}{$\begin{array}{l}\text { Web and mobile applications that serve as platforms for social } \\
\text { networking }\end{array}$} & Discussion forums for patients on dialysis \\
\hline & & Promotion of donor registration and finding living kidney donors \\
\hline
\end{tabular}

The listing of categories and applications is not comprehensive; many eHealth interventions are multimodal and definitions may overlap. AKI, acute kidney injury; app, application; CKD, chronic kidney disease; PD, peritoneal dialysis.

across eight centres to either usual care or a multimodal adherence intervention and used an electronic pillbox to monitor adherence. In the intervention group, participants reviewed their own adherence data with a coach, identified barriers to adherence that they would like to address and used problem-solving methods with the coach to find solutions. Participants also chose how to receive medication reminders, either by text message, email or visual cues. The TAKE-IT intervention significantly improved medication adherence compared with the control group. This finding demonstrates that involving patients as stakeholders to individualize eHealth interventions and meet patient needs can improve outcomes.

\section{Challenges for adopting eHealth}

A number of challenges to adopting eHealth interventions in kidney care exist. First, although eHealth can enhance disease detection and monitoring, it can introduce new safety concerns owing to suboptimal design, technological failures or invalid data. For example, the study of mobile apps discussed above found that only two of seven health tracking apps generated alerts for abnormal values such as extremely high blood pressure $^{7}$. Device failures in clinical trials have resulted in reduced participant satisfaction, engagement and retention with eHealth monitoring ${ }^{6}$. Cuff-less blood pressure devices based on pulse transit time have been found to correlate with oscillometric measurements in research settings, but the accuracy depends on posture and the devices require frequent calibration ${ }^{9}$. Many wearable devices will require additional validation before clinical use. Clinicians and patients must remain vigilant about the potential for errors, particularly if eHealth tools are used in place of traditional monitoring without full validation.

Second, patients with kidney disease have complex needs that can make remote monitoring via eHealth tools less suitable than traditional face-to-face care. Certain aspects of the physical exam, such as examination of catheter exit sites, may be difficult to perform via video consultation. The physical presence of the 
provider may also be reassuring and comforting for patients with emotional distress ${ }^{4,5}$. Providing CKD education via web modules, mobile apps or text messaging may not fully address the complexity of kidney care or patient-specific questions. Thus, the US 2018 Bipartisan Budget Act allows patients to choose between telehealth and face-to-face encounters so that the care modality meets their individual needs ${ }^{5}$.

Other issues exist that are not unique to kidney care. Implementing eHealth interventions requires resource reallocation that may result in substantial upfront investment. Technological infrastructure and adequate security measures must be in place. For example, telehealth dialysis visits require equipment such as webcams and digital stethoscopes as well as access to a reliable internet service with sufficient speed to enable real-time video transmission. All hardware and software tools must meet relevant security standards, and patients and care providers must be trained to use the equipment correctly and conduct the visits securely ${ }^{5}$. An ongoing extension of the TAKE-IT study, TAKE-IT TOO, aims to address the practical issues of incorporating the TAKE-IT intervention into routine clinical practice ${ }^{8}$. In addition, the NIDDK is developing a financial model to help health-care organizations plan investments in health information technology infrastructure to support CKD management (see Related links).

\section{The road ahead}

Despite the currently limited evidence of efficacy and numerous challenges, eHealth holds tremendous promise for patient-centred disease management in nephrology. Moving forwards, patient engagement will be critical to the development of pragmatic and relevant interventions to provide a care continuum from clinical settings to the patient's home. Hybrid effectiveness-implementation or pragmatic and adaptive trial designs that simultaneously address feasibility, implementation and efficacy may be appropriate and efficient ways of evaluating novel eHealth solutions. The COVID-19 pandemic highlights the importance of addressing current evidence gaps in eHealth.

1. International Telecommunication Union. Facts and Figures 2019 Measuring Digital Development. https://itu.foleon.com/itu measuring-digital-development (ITU, 2019).

2. Eysenbach, G. What is e-health? J. Med. Internet Res. 3, e20 (2001).

3. Norton, J. M. et al. Development and validation of a pragmatic electronic phenotype for CKD. Clin. J. Am. Soc. Nephrol. 14 1306-1314 (2019).

4. Crowley, S. T. et al. Targeting access to kidney care via telehealth the VA experience. Adv. Chronic Kidney Dis. 24, 22-30 (2017).

5. Lew, S. Q. $\&$ Sikka, N. Operationalizing telehealth for home dialysis patients in the United States. Am. J. Kidney Dis. 74, 95-100 (2019).

6. Stevenson, J. K. et al. eHealth interventions for people with chronic kidney disease. Cochrane Database Syst. Rev. 8, CD012379 (2019).

7. Foster, B. J. et al. A randomized trial of a multicomponent intervention to promote medication adherence: the Teen Adherence in Kidney Transplant Effectiveness of Intervention Trial (TAKE-IT). Am. J. Kidney Dis. 72, 30-41 (2018).

8. Singh, K. et al. Patients' and nephrologists' evaluation of patientfacing smartphone apps for CKD. Clin. J. Am. Soc. Nephrol 14, 523-529 (2019).

9. Park, S. H., Zhang, Y., Rogers, J. A. \& Gallon, L. Recent advances of biosensors for hypertension and nephrology. Curr. Opin. Nephrol. Hypertens. 28, 390-396 (2019)

\section{Acknowledgements}

C.W. is supported by the National Institute of Diabetes and Digestive and Kidney Diseases of the National Institutes of Health (NIH) under Award Number K23DK118189. The content is solely the responsibility of the authors and does not necessarily represent the official views of the NIH. The authors thank L. Greenbaum (Emory University and Children's Healthcare of Atlanta, Atlanta, GA, USA) for his input.

Competing interests

The authors declare no competing interests.

\section{RELATED LINKS}

NKDEP Health Information Technology Working Group: https://www.niddk. nih.gov/health-information/communication-programs/nkdep/working-groups/ health-information-technology 